\title{
TEN AXIOMS FOR THREE DIMENSIONAL EUCLIDEAN GEOMETRY
}

\section{LINO GUTIERREZ NOVOA}

1. Introduction. The notion of an $n$-ordered set, introduced in [3], allows a drastic reduction in the number of axioms usually employed to characterize Euclidean 3-geometry. (This number varies for the different systems: Hilbert, 21; Veblen-Hilbert, 18; Borsuk-Szmielew, 27 ; Huntington, 26. The so-called metric systems involve the notion of real number which requires several additional axioms for its definition.)

The simplification carried out in this paper is possible because two of the axioms, $\mathrm{P}_{1}$ and $\mathrm{P}_{2}$, have deep consequences which condense almost all of the usual notions of incidence and order of other systems.

The questions concerning independence of the axioms are not touched here. Also left aside are the interesting pedagogical possibilities of the system.

The generalization to $n$-dimensions seems to be straightforward.

2. The axioms. The first four axioms characterize a notion of 3 -order on a set $\varepsilon$. This means there is a relation defined on the set of tetrads (ordered sequences of four points of $\varepsilon$ ) which satisfies $P_{1}$ through $\mathrm{P}_{4}$. It is more convenient to define this relation using a characteristic function $\phi$, which is defined on $\varepsilon \times \varepsilon \times \mathcal{E} \times \mathcal{E}$ and takes values in the set $\{-1,0,1\}$. For brevity we write:

$$
\phi\left(A_{1}, A_{2}, A_{3}, A_{4}\right)=\left\langle A_{1}, A_{2}, A_{3}, A_{4}\right\rangle \quad \text { for } A_{i} \in \varepsilon .
$$

(The reader is referred to [3] for the basic definitions and results.) $\mathrm{P}_{1}$. If $A_{i} \in \mathcal{E}, i=1,2,3,4$, then:

$$
\left\langle A_{1}, A_{2}, A_{3}, A_{4}\right\rangle=\left\langle A_{2}, A_{3}, A_{4}, A_{1}\right\rangle=-\left\langle A_{2}, A_{1}, A_{3}, A_{4}\right\rangle .
$$

$\mathrm{P}_{1}$ implies that $\phi$ is defined on the classes of ordered tetrads. Two tetrads being equivalent if they are permutations of the same type.

$\mathrm{P}_{2}$ (Exchange AXIOM). If $\left\langle B_{1}, A_{2}, A_{3}, A_{4}\right\rangle\left\langle A_{1}, B_{2}, B_{3}, B_{4}\right\rangle$, $\left\langle B_{2}, A_{2}, A_{3}, A_{4}\right\rangle\left\langle B_{1}, A_{1}, B_{3}, B_{4}\right\rangle,\left\langle B_{3}, A_{2}, A_{3}, A_{4}\right\rangle\left\langle B_{1}, B_{2}, A_{1}, B_{4}\right\rangle$, and $\left\langle B_{4}, A_{2}, A_{3}, A_{4}\right\rangle\left\langle B_{1}, B_{2}, B_{3}, A_{1}\right\rangle$ are all nonnegative then:

$$
\left\langle A_{1}, A_{2}, A_{3}, A_{4}\right\rangle\left\langle B_{1}, B_{2}, B_{3}, B_{4}\right\rangle \geqq 0 .
$$

In this axiom, one element, $A_{1}$, of the first tetrad, is interchanged

Received by the editors November 4, 1966. 
with all the elements of the second tetrad, maintaining the order. The element $A_{1}$ has no special significance, since, by $\mathrm{P}_{1}$, any element can be brought to occupy the first place.

$\mathrm{P}_{3}$. The 3-order of $\varepsilon$ is nontrivial, i.e.: for some tetrad $\left\langle A_{1}, A_{2}, A_{3}, A_{4}\right\rangle$ $\neq 0$.

$\mathrm{D}_{1}$. The point $A_{1}$, is said to be singular if for every tetrad which contains it:

$$
\left\langle A_{1}, A_{2}, A_{3}, A_{4}\right\rangle=0 .
$$

Analogous definitions for singular pairs and triples. In particular, a tetrad $\left(A_{i}\right)$ is singular if $\left\langle A_{1}, A_{2}, A_{3}, A_{4}\right\rangle=0$.

$\mathrm{D}_{2}$. Given a nonsingular pair $(A, B)$ the line $A B$ is the set $l(A, B)$ $=\{X ;(A, B, X)$ is singular $\}$.

Given a nonsingular triple $(A, B, C)$, the plane $A B C$ is the set $\pi(A, B, C)=\{X ;(A, B, C, X)$ is singular $\}$.

$\mathrm{D}_{3} . \pi(A, B, C)$ is said to separate $X$ and $Y$ if

$$
\langle A, B, C, X\rangle \neq\langle A, B, C, Y\rangle \text {. }
$$

$\mathrm{P}_{4}$. If the pair $(A, B)$ is singular, then $A=B$.

$\mathrm{P}_{5}$. If $\pi(A, B, C)$ separates $X$ and $Y$, then

$$
\pi(A, B, C) \cap l(X, Y) \neq 0 .
$$

Our sixth axiom is the parallel's axiom in Playfair's form.

$\mathrm{P}_{6}$ (PARALlel's AXIOM). If $P \notin l(A, B)$ there is one unique line $l^{\prime}$ in $\pi(A, B, P)$ which does not meet $l(A, B)$. ( $l^{\prime}$ is said to be parallel to $\left.l.\right)$

$\mathrm{D}_{4}$. Given a point $P$ not in the plane $\pi(A, B, C)$ one relative 2-order of $\pi(A, B, C)$ is defined by

$$
\langle X, Y, Z\rangle=\langle P, X, Y, Z\rangle \text { for } X, Y, Z \in \pi \text {. }
$$

In [3] it was proved that this notion is independent of $P$ save for reversings of the order and that there are two natural induced 2orders on every $\pi \in \mathcal{E}$.

In a similar manner, the 1 -orders on the lines are defined. Given $Q$, not on $l$, we put: $\langle X, Y\rangle=\langle Q, X, Y\rangle$, for $X, Y \in l$. Again this notion is independent of the point $Q$ and there are two natural 1-orders on every line.

$\mathrm{D}_{5}$. An upper bound $K$ of a set $\left\{X_{\alpha}\right\} \subset l$ for the relative 1-order $\langle$,$\rangle of the line l$, is a point $K$ which satisfies: $\left\langle K, X_{\alpha}\right\rangle \geqq 0$ for every $\alpha$. Similar definition for lower bound.

A least upper bound of a subset $\delta$ of $l$ is a lower bound of the set of all upper bounds of $S$ which is itself an upper bound of $\delta$.

$\mathrm{P}_{7}$. Every line of $\mathcal{E}$ is conditionally complete in any of its relative 
orders i.e.: every subset of it which has an upper bound has also a least upper bound.

As will be proved in the sequel, the above axioms characterize the real affine space of three dimensions. It only remains to add suitable axioms to especialize to Euclidean geometry. One way to accomplish this would be to add congruence axioms, but since our goal is to keep the number of axioms at a minimum, we prefer to introduce, with our last three axioms, the notion of "orthogonality" between a line $l$ and a plane $\pi$, denoted by $l \perp \pi$. A definition and some notation is needed.

$\mathrm{D}_{6}$. A plane is said to be parallel to a line if they do not intersect.

The symbol $l / / \pi$ will be used to mean that either 1 is parallel to $\pi$ or $l \subset \pi$. Similarly $l / / l^{\prime}$ means that $l$ is identical or parallel to $l^{\prime}$. Now the final axioms:

$\mathrm{P}_{8}$. If $l \perp \pi$ then $l / / \pi$ is not true.

$\mathrm{P}_{9}$. Given a point, $P$, and a plane, $\pi$, then, for some line $l: l \perp \pi$, and $P \in l$.

$\mathrm{P}_{10}$. If $l \perp \pi$ and $l^{\prime} \perp \pi^{\prime}$ then $l / / \pi^{\prime} \Rightarrow l^{\prime} / / \pi$.

3. The incidence structure. We prove, in first place, that Hilbert's connection (incidence) axioms hold in our system. (See $[2$, p. 4].) Let $\varepsilon$ be a 3 -ordered set satisfying $\mathrm{P}_{1}$ through $\mathrm{P}_{10}$. The definition of $l(A, B)$ in the previous section, together with $\mathrm{P}_{4}$, show that there is one line through $A$ and $B$. The uniqueness of this line, and the fact that any two distinct points of it determine the same line, follow from the following theorem:

$\mathrm{T}_{1}$. Let $C \neq D$ be on $l(A, B)$. Then, $l(A, B)=l(C, D)$.

Proof. Let $X \in l(A, B)$ and consider the triples $(X, C, D)$ and $(A, B, Q)$ where $Q \notin l(A, B)$. In any relative order of $\pi(A, B, Q)$ apply $\mathrm{P}_{2}$ (exchanging $Q$ ) to show $\langle X, C, D\rangle\langle A, B, Q\rangle=0$. Hence $\langle X, C, D\rangle=0$ or equivalently, $X \in l(C, D)$. Similarly for the opposite inclusion. Notice that we use $\mathrm{P}_{3}$ implicitly in this proof.

In a similar way, the existence and uniqueness of a plane through three points is established. Also the fact that any three noncollinear points on a plane determine it.

That a line which has two points on a plane is contained in it, follows from our definitions of lines and planes. Also these definitions, together with $\mathrm{P}_{3}$, imply that there are at least two points on every line, three noncollinear points on every plane and four noncoplanar points in $\mathcal{E}$.

So, it only remains for Hilbert's I, 6 of [2] to be proved. This axiom reads:

$\mathrm{T}_{2}$. If two planes $\alpha, \beta$, have a point $A$ in common, then they have at least a second point in common. 
To prove this we need some preparation. First we show that axiom $\mathrm{P}_{5}$ holds in the planes with their relative orders.

$\mathrm{T}_{3}$. In any relative order of a plane $\pi$, if

$$
\left\langle A_{1}, A_{2}, X\right\rangle \neq\left\langle A_{1}, A_{2}, Y\right\rangle \text { then } l(X, Y) \cap l\left(A_{1}, A_{2}\right) \neq 0 .
$$

Proof. The given inequality means that for some $P \in \pi$ $\left\langle P, A_{1}, A_{2}, X\right\rangle \neq\left\langle P, A_{1}, A_{2}, Y\right\rangle$. Hence, by $\mathrm{P}_{5}, l(X, Y)$ meets $\pi\left(A_{1}, A_{2}, P\right)$. But $\pi \cap \pi\left(A_{1}, A_{2}, P\right)=l\left(A_{1}, A_{2}\right)$ and this proves the theorem.

$\mathrm{C}_{1}$. If $l(A, B) / / l^{\prime}$, then for any $X, Y \in l^{\prime}$ :

$$
\langle A, B, X\rangle=\langle A, B, Y\rangle \text {. }
$$

$\mathrm{C}_{2}$. Given four points $A, B, X, Y$ in a plane $\pi$. If $l(A, B) / / l(X, Y)$ and $l(A, X) / / l(B, Y)$ then:

$$
\langle A, B, X\rangle=\langle X, B, Y\rangle \text {. }
$$

Proof. By the preceding corollary:

$$
\langle A, B, X\rangle=\langle A, B, Y\rangle=\langle X, B, Y\rangle
$$

$\mathrm{T}_{4}$. Let $\pi=\pi(A, B, Z)$ meet line $l=l(Z, M)$ in $Z$ and $l \nsubseteq \pi$. Then there are at least two points $X$ and $Y$ in $l$ such that:

$$
\langle X, A, B, Z\rangle=-\langle Y, A, B, Z\rangle \neq 0 \text {. }
$$

Proof. Consider the parallels to $l(M, A)$ and to $l(M, Z)$ through $Z$ and $A$ respectively. Let them meet at $T$. Then by $\mathrm{C}_{2}:\langle M, A, Z\rangle$ $=\langle Z, A, T\rangle \neq 0$ in the ordered plane $\pi(M, A, Z)$. The parallel to $A Z$ through $T$ will meet $l$ at a point $Y$ and by $\mathrm{C}_{1}:\langle Z, A, T\rangle=\langle Z, A, Y\rangle$ and therefore: $\langle M, A, Z\rangle=\langle Z, A, Y\rangle$. It follows that for any point, say $B$ :

$$
\langle M, A, B, Z\rangle=\langle Z, A, B, Y\rangle=-\langle Y, A, B, Z\rangle \neq 0 .
$$

Putting $X=M$ we get the theorem.

Now we can prove Hilbert's I, 6:

$\mathrm{T}_{5}$. If the two planes $\pi(Z, B, C)$ and $\pi^{\prime}(Z, N, P)$ have a common point $Z$, they have another one, $W$.

Proof. On $l(Z, B)$ and $l(Z, C)$ take four points $X, Y, X^{\prime}, Y^{\prime}$, furnished by the previous theorem, so that:

$$
\begin{aligned}
& \langle X, Z, N, P\rangle=-\langle Y, Z, N, P\rangle \neq 0 ; X, Y, \in l(Z, B) \subset \pi(Z, B, C), \\
& \left\langle X^{\prime}, Z, N, P\right\rangle=-\left\langle Y^{\prime}, Z, N, P\right\rangle \neq 0 ; X^{\prime}, Y^{\prime}, \in l(Z, C) \subset \pi(Z, B, C) .
\end{aligned}
$$

Choose a pair from those four points, say $U, V$, which belong to different lines, and such that: 


$$
\langle U, Z, N, P\rangle=-\langle V, Z, N, P\rangle \neq 0 .
$$

By $\mathrm{P}_{5}, l(U, V) \cap \pi(Z, N, P) \neq 0$. Let $X$ be a point in that intersection. Then $X$ is clearly different from $Z$, otherwise $Z, B, C$ would be collinear. The proof is complete.

We have thus proved that all the axioms of connection of the Hilbert system are valid in $\varepsilon$.

Theorem $\mathrm{T}_{4}$ implies the existence of points on $l(P, Q)$ exterior to the segment $P Q$ as the following corollary shows:

$\mathrm{C}_{3}$. On the line $l=l(P, Q)$ there is a point $R$ such that $\langle P, Q\rangle$ $=\langle Q, R\rangle$.

Proof. Take a plane $\pi(Q, A, B)$ which does not contain $l$ (the existence of it is guaranteed by $\left.\mathrm{P}_{3}\right)$ and apply $\mathrm{T}_{4}$.

We can also prove the existence of a point $S \in l$ interior to $P Q$ i.e.: $\langle P, S\rangle=\langle S, Q\rangle$. For this case we use one of the forms of Desargues' theorem, which holds in any plane $\pi$ of $\mathcal{E}$ because its proof depends on the fact that $\pi$ is imbedded in $\mathcal{E}$.

We show first:

$T_{6}$. If $P L Q M$ is a parallelogram (in an obvious sense) and $R=l(P, Q)$ $\cap l(L, M)$ then: $\langle P, R\rangle=\langle R, Q\rangle$. (The existence of $R$ is guaranteed by $\mathrm{T}_{3}$ and $\mathrm{C}_{2}$.)

Proof. Let $L S / / P Q$ and $S Q / / L M$. Then (by Desargues) it follows that $R S / / P L$. Applying $\mathrm{C}_{2}$ :

$$
\langle P, R, L\rangle=\langle R . S, L\rangle=\langle L, Q, S\rangle=\langle R, Q, L\rangle
$$

or $\langle P, R, L\rangle=\langle R, Q, L\rangle$ which proves the theorem.

$\mathrm{T}_{7}$. Given $P$ and $Q$, there is some $R \in l(P, Q)$ such that $\langle P, R\rangle$ $=\langle R, Q\rangle$.

Proof. Obviously, a parallelogram can be constructed with $P Q$ as a diagonal. Then apply $\mathrm{T}_{6}$.

4. Imbedding in the real projective 3-space. The easiest way to complete the proof is as follows. We imbed $\varepsilon$ into a space $\rho$ by the well-known method of adjoining the ideal elements to $\mathcal{E}$. This induces an incidence structure on $P$. A separation order is then defined on $P$ and it is shown that, under these conditions, $P$ is the real projective 3 -space. Finally it is proved that the orthogonality axioms define an elliptic polarity on the ideal plane.

We shall give only a sketch of this proof, leaving the details to the reader.

Assume we add to $\mathcal{E}$ the set $\mathscr{g}$ of ideal points (i.e. maximal families of parallel lines) to get $P=\varepsilon \cup \mathscr{g}$. It is well known that, in our case, with the usual definitions, $\beta$ is a Desarguesian projective 3 -space. 
We take any categorical axiom system for the real projective 3 -space, for instance [1, pp. 20-23]. The incidence axioms are automatically satisfied and need not be checked.

$\mathrm{D}_{7}$. The separation order-function on a line $l$ of $\varepsilon$ is the function: $\sigma(A, B, C, D)=\langle A, C\rangle\langle A, D\rangle\langle B, C\rangle\langle B, D\rangle$ where $A, B, C, D$ are four distinct points on the line $l$ and $\langle$,$\rangle denotes any relative order$ of $l$. (It is clear that this function is independent of which order we use.) This function is extended to the ordinary lines of $\rho$ by defining:

$$
\begin{aligned}
\sigma(A, B, C, \Omega) & =\sigma(A, B, \Omega, C)=\sigma(C, \Omega, A, B)=\sigma(\Omega, C, A, B) \\
& =\langle A, C\rangle\langle B, C\rangle
\end{aligned}
$$

where $\Omega$ is the ideal point of $l$.

To extend it to the ideal lines we observe first:

$\mathrm{T}_{8}$. If $A, B, C, D$ are distinct points on the line $l \in \mathcal{E}, Q$ any point in $-l$, and $X$ any point on $\mathcal{E} \cap l(Q, A)$ different from $Q$ then:

$$
\sigma(A, B, C, D)=\langle Q, X, C\rangle\langle Q, B, C\rangle\langle Q, X, D\rangle\langle Q, B, D\rangle
$$

where $\langle, \quad\rangle$ is some relative order of $\pi(A, l)=\pi$.

Proof. Taking the order of $l$ relative to $Q$ we see that: $\sigma(A, B, C, D)$ $=\langle Q, A, C\rangle\langle Q, B, C\rangle\langle Q, A, D\rangle\langle Q, B, D\rangle$.

Now apply $\mathrm{P}_{2}$ to the triples $(Q, A, C),(Q, X, D)$ and, exchanging $C$, we have: $\langle Q, A, C\rangle\langle Q, A, D\rangle=\langle Q, X, C\rangle\langle Q, X, D\rangle$. Multiplying both sides by $\langle Q, B, C\rangle\langle Q, B, D\rangle$ the result follows.

$\mathrm{C}_{4}$. The above theorem is true if $A=\Omega$.

Proof. Since $\langle Q, X, D\rangle\langle Q, X, C\rangle=1$, by $C_{1}$, we have, in this case, $\sigma(\Omega, B, C, D)=\langle B, C\rangle\langle B, D\rangle=\langle Q, X, C\rangle\langle Q, B, C\rangle\langle Q, X, D\rangle\langle Q, B, D\rangle$.

$\mathrm{D}_{8}$. Given four concurrent lines $l_{i}$ on a plane $\pi$, we define the function $\sigma^{\prime}\left(l_{1}, l_{2}, l_{3}, l_{4}\right)=\sigma\left(L_{1}, L_{2}, L_{3}, L_{4}\right)$, where $L_{i}$ is the intersection of $l_{i}$ and some line $l_{0}$. In view of the preceding corollary, this definition makes sense, even if one of the lines is ideal. That it also makes sense if the lines meet on some ideal point (are parallel) follows from the following theorem:

$\mathrm{T}_{9}$. Every parallel projection between two lines is an (relative) orderpreserving or order-reversing transformation.

Proof. Is left to the reader.

$D_{9}$. Given four collinear ideal points $\Omega_{i}, i=1,2,3$, 4, we define $\sigma\left(\Omega_{1}, \Omega_{2}, \Omega_{3}, \Omega_{4}\right)=\sigma^{\prime}\left(l_{1}, l_{2}, l_{3}, l_{4}\right)$ where $l_{i}$ are concurrent lines such that $\Omega_{i} \in l_{i}$.

That this definition is independent of the point $Q$ where the lines meet is not difficult to prove.

Now we can check that the order axioms of [1] hold in $P$ if we define: 


$$
A B / C D \text { to mean } \sigma(A, B, C, D)=-1 .
$$

We omit the details.

Since our continuity axiom is equivalent to 2.13 of [1] the proof that $P$ is the real projective 3 -space is now complete.

It is well known that Euclidean 3-space can be obtained from the real projective 3 -space $P^{3}$ by selecting a plane $\pi \subset P^{3}$ and defining one imaginary conic $\Gamma$ (the absolute) on that plane. (See $[4$, pp. 289292].) The notion of orthogonality between planes and lines can then be introduced in $P^{3}-\Gamma$; a line being orthogonal to a plane if their intersections with $\pi$ are pole and polar with respect to the elliptic polarity defined by $\Gamma$. The group of $E^{3}=P^{3}-\Gamma$ is the subgroup of projectivities of $P^{3}$ which leave $\Gamma$ invariant, or equivalently, those which preserve the relation of orthogonality mentioned above.

That our last three axioms $\mathrm{P}_{8}, \mathrm{P}_{9}$ and $\mathrm{P}_{10}$ define an elliptic polarity on the ideal plane of $\mathcal{E}$ will now be shown.

The first step is to prove that the notion of orthogonality is invariant under parallelism, i.e. $l \perp \pi, l / / l^{\prime}$ and $\pi / / \pi^{\prime}$ imply $l^{\prime} \perp \pi^{\prime}$. This follows rather easily from the axioms.

Let $\pi_{0}$ be the ideal plane of $\mathcal{E}$. Given any ideal point $\Omega \in \pi_{0}$ we may use $\mathrm{P}_{9}$ to define a function $\tau(\Omega)$ which takes values on the set of ideal lines of $\pi_{0}$ in the following way: $\tau(\Omega)=\omega$ if $l \perp \pi$ where $l$ is any ordinary line incident with $\Omega$ and $\pi$ any ordinary plane incident with $\omega$. Because of the previous remark $\tau$ is a bijective function which maps the points of $\pi_{0}$ onto the lines of $\pi_{0}$. Furthermore, axiom $\mathrm{P}_{10}$ guarantees that collinear points go into concurrent lines and that $\tau^{-1}$ has a similar property. Thus $\tau$ is a polarity defined on $\pi_{0}$, which is elliptic because of $\mathrm{P}_{8}$. This completes the proof that the structure defined in $\mathcal{E}$ by our ten axioms is equivalent to the usual geometric structure of $E^{3}$.

\section{REFERENCES}

1. H. S. M. Coxeter, Non Euclidean geometry, 3rd ed., Univ. of Toronto Press, Toronto, 1957.

2. D. Hilbert, The foundations of geometry, Open Court, La Salle, Illinois 1959.

3. L. G. Novoa, On n-ordered sets and order completeness, Pacific J. Math. 15 (1965), 1337-1345.

4. C. E. Springer, Geometry and analysis of projective spaces, Freeman, San Francisco, Calif., 1964.

University of Alabama 